\title{
Predictores de las actitudes hacia la inclusión de alumnado con necesidades educativas especiales en futuros profesionales de la educación
}

\section{Predictors of attitudes toward inclusion of students with special educational needs in future education professionals}

José Luis ÁLVAREZ CASTILLO y Mariana BUENESTADO FERNÁNDEZ Universidad de Córdoba

Recibido: Febrero 2014

Aceptado: Mayo 2014

\section{Resumen}

Uno de los principales pilares en la construcción de escuelas inclusivas es la formación inicial del profesorado. Antes de determinar si es necesario realizar cambios y de qué tipo en los programas formativos o planes de estudios en relación con la atención a la diversidad y con la educación inclusiva, deben analizarse, a modo de diagnóstico de necesidades, las actitudes que presentan los futuros profesionales de la educación al respecto, incluyendo la identificación de predictores relevantes. La investigación sobre la que se informa en este trabajo persiguió esta finalidad, y lo hizo con una metodología cuantitativa basada en encuesta, a través del uso de instrumentos estructurados de recogida transversal de información y de análisis estadísticos relacionados con la calidad del cuestionario empleado para medir las actitudes (análisis factorial y alpha de Cronbach), análisis descriptivos y correlacionales, pruebas de hipótesis sobre diferencia de medias actitudinales, y análisis de regresión lineal para identificar los predictores de las actitudes hacia la inclusión. Los resultados muestran, en primer lugar, que los participantes se caracterizan por poseer actitudes positivas hacia la inclusión de personas con necesidades educativas especiales, especialmente los estudiantes mayores y de cursos más avanzados y, en menor medida, las mujeres y quienes han tenido contacto previo con estas personas. En segundo lugar, se evidencia que los valores de autotrascendencia y, más débilmente, el contacto funcionan como predictores robustos de las actitudes de los futuros profesionales de la educación hacia la inclusión de este tipo de alumnado. En la discusión se establecen algunas aplicaciones para la profesionalización de los futuros educadores.

Palabras clave: educación inclusiva, actitudes, necesidades educativas especiales, formación inicial del profesorado, predicción.

\begin{abstract}
One of the main pillars in the development of inclusive schools is the initial teacher training. Before determining if it is necessary to make changes (and of what type) in training programs or curriculum guides related to the attention to diversity and inclusive education, the attitudes of future education professionals in this area should be analyzed. This includes the identification
\end{abstract}


of the relevant predictors of inclusive attitudes. The research reported in this article pursued this objective, doing so with a quantitative survey methodology based on the use of cross-sectional structured data collection and statistical analyses related to the quality of the attitude questionnaire (factor analysis and Cronbach's alpha), descriptive statistics, correlations, hypothesis tests for difference of means, and regression analysis in order to predict attitudes towards inclusion in education. Firstly, the results show that the participants held very positive attitudes toward the inclusion of students with special educational needs. Particularly, older respondents, those with a longer training and, to a lesser extent, women and those who had been in touch with disabled people stood out within this attitude. Secondly, it is evidenced that selftranscendence values and, more weakly, contact, function as robust predictors of attitudes of future practitioners towards the inclusion of students with special needs. Some applications for the initial professionalization of educators are suggested in the discussion.

Keywords: inclusive education, attitudes, special needs students, preservice teacher education, prediction.

La promoción de la calidad de la educación inclusiva requiere una formación inicial y continua del profesorado basada en conocimientos, competencias, valores y actitudes, vinculándose todos estos elementos al principio de justicia social y alejándose del modelo del déficit. Esta dimensión formativa resulta crucial para el desarrollo de la inclusión (Agencia Europea para el Desarrollo de la Educación del Alumnado con Necesidades Educativas Especiales, 2009, 2010). Particularmente, en relación con las actitudes se sabe que el profesorado que posee una predisposición más favorable hacia la inclusión de alumnado con necesidades educativas especiales (NEE), es aquel que también dispone de más estrategias educativas para que la inclusión tenga éxito (Ahmmed, Sharma y Deppeler, 2012; Avramidis y Norwich, 2002; Chiner, 2011; Swain, Nordness y Leader-Janssen, 2012). Asimismo, la relación entre la autoeficacia y las actitudes empieza a evidenciarse en la formación inicial del profesorado (Gao y Mager, 2011; Loreman, Sharma y Forlin, 2013) y se mantiene robusta luego en la etapa en ejercicio (Savolainen, Engelbrecht, Mirna y Malinen, 2012), lo que aún justifica en mayor medida la necesidad de promover las actitudes positivas hacia la inclusión en la formación (Selesho, 2012; Taylor y Ringlaben, 2012).

En general, se podrían etiquetar de heterogéneos los estudios realizados para conocer las actitudes inclusivas en relación con alumnado con NEE. Dos de las fuentes de variación son el tipo de participantes y el de variables medidas que potencialmente se asocian a las actitudes. En cuanto a las muestras, predominan los profesionales de la educación en activo. Boer, Pijl y Minnaert (2011), a partir de la revisión de 26 estudios en revistas internacionales, dejaron patente que la mayoría del profesorado mantiene actitudes neutrales o negativas hacia la inclusión de este alumnado en clases ordinarias de educación primaria. Estos profesionales presentan, del mismo modo, una serie de preocupaciones relacionadas con la cantidad de tiempo empleado con el alumnado con necesidades especiales, la falta de servicios de apoyo y preparación para dicha práctica, y la insatisfacción por la baja calidad de sus trabajos, y también por otra serie de efectos negativos asociados a la inclusión (Tait y Purdie, 2000; Hwang y Evans, 2011). En otras investigaciones no se han hallado tales actitudes negativas hacia la inclusión, 
pero sí las preocupaciones relacionadas con la necesidad de apoyos, que a su vez dependen de las actitudes previas (Mangano, 2015; Monsen, Ewing y Kwoka, 2014).

Un segundo tipo de muestra es la de futuros profesionales de la educación, quienes se manifiestan normalmente favorables a la inclusión. A pesar de que los predictores actitudinales no se encuentran perfectamente identificados, se dispone de estudios internacionales (e.g., Forlin, Sharma y Loreman, 2007; Gao y Mager, 2011; Kim, 2011; Swain et al., 2012) y nacionales (e.g., Tárraga, Grau y Peirat, 2013) que demuestran que las actitudes hacia la inclusión son alteradas cuando entran en juego variables de formación o de experiencia con personas con NEE.

En lo que se refiere a la formación, la adquirida durante la etapa inicial es clave en el desarrollo de actitudes positivas. Investigaciones como las de Sze (2009) evidencian que los futuros profesionales de la educación están de acuerdo con la educación inclusiva, pero se sienten poco preparados para poder satisfacer las necesidades de un alumnado diverso dentro del aula. Para acometer esta tarea, la formación del pensamiento práctico es sumamente relevante (Pérez Gómez, 2010), y esta se puede llevar a cabo con actividades que combinen la teoría con la práctica de campo (Swain et al., 2012). Aunque diferentes currículum de formación de profesorado puedan incidir diferencialmente en las actitudes hacia la inclusión (Kim, 2011), lo cierto es que los contenidos de los programas de formación inicial relacionados con la educación inclusiva han aumentado durante los últimos años (Gallego y Rodríguez, 2012).

La investigación sobre la predicción de las actitudes inclusivas no queda restringida, sin embargo, a los factores formativos. Así, por ejemplo, se ha demostrado que el contacto o experiencia previa con personas con NEE facilita las actitudes y experiencias inclusivas en profesorado en ejercicio (Ahmmed et al., 2012; Avramidis, Bayliss y Burden, 2000). Lo mismo ocurre en los futuros profesionales de la educación, especialmente en aquellos que han recibido una instrucción formal al mismo tiempo que han desarrollado prácticas educativas en entornos inclusivos (Campbell, Gilmore y Cuskelly, 2003; McCollow, Shurr y Jasper, 2015; Swain et al., 2012). Además, no solo es importante la mera existencia de experiencia relacional previa, sino que la literatura psicosocial sobre la denominada "hipótesis del contacto" ha mostrado que la frecuencia y calidad de esta relación también incide en la evolución de las actitudes (Allport, 1954; Hewstone y Swart, 2011; Pettigrew y Tropp, 2011), luego la duración y el tipo del contacto son variables que asimismo deben ser consideradas en la investigación educativa. Por ejemplo, Rodríguez, Etopa y Rodríguez (2002) y de Polo, Fernández y Díaz (2011) ya han desvelado que los estudiantes con actitudes más positivas hacia la inclusión son los que cuentan con experiencia previa con personas con NEE en un ámbito específico: el familiar.

Por su parte, el estudio de valores humanos, conceptuados estos como entidades cognitivas que actúan como criterios y guían la selección de acciones, también tienen cierto interés, junto con la formación y el contacto, en el ámbito de la predicción de las actitudes. Según Luque y Luque-Rojas (2011), las creencias del profesorado acerca del alumnado con NEE se relacionan con los valores y actitudes sociales. En esta línea, se ha evidenciado que los estudiantes de magisterio que defienden el derecho que tienen 
todos los niños a ser educados sin exclusiones manifiestan valores de justicia, igualdad y colaboración (Sandoval, 2009).

De acuerdo con el marco teórico presentado, el primer propósito de nuestro estudio, basado en encuesta con recogida transversal de datos, es conocer las actitudes de los estudiantes de magisterio y psicopedagogía hacia la inclusión de alumnado con NEE. En segundo lugar, se pretende comprobar los vínculos que mantienen estas actitudes con variables sociodemográficas (edad, sexo), formativas (titulación, curso), de experiencia previa con personas con NEE (incluyendo el tipo de contacto y la duración del mismo), y con los valores que propone Schwartz (1992, 2012) en su Teoría de Valores Humanos Básicos: poder, logro, hedonismo, estimulación, autodirección, universalismo, benevolencia, tradición, conformidad y seguridad. Se ha seleccionado esta teoría de valores por su consistencia y universalismo, pero también por relacionar los valores con las motivaciones básicas, acercándolos por tanto a la conducta (particularmente, la dimensión de autotrascendencia vs. autopromoción se halla asociada a las motivaciones de inclusión/exclusión [Basabe, Valencia y Bobowik, 2011]).

En relación con la formulación de las hipótesis, se plantea en primer lugar que conforme los futuros docentes vayan adquiriendo competencias en educación inclusiva y atención a la diversidad, sus actitudes tenderán también a ser más positivas (i.e., la edad, el nivel de la titulación y el curso covariarán positivamente con las actitudes). Asimismo, es probable que se verifique un vínculo de sentido positivo entre las actitudes inclusivas y la experiencia previa con personas con NEE (i.e., relación personal de duración variable con personas con NEE en ámbitos formativos, laborales, familiares o, en general, sociales de diverso tipo -asociacionismo, vecindad, espacios de ocio y tiempo libre-), la duración de la misma y los valores de tendencia aperturista (i.e., valores que enfatizan la independencia personal y la disponibilidad para el cambio: autodirección y estimulación) y de autotrascendencia (i.e., valores que enfatizan el interés por el bienestar de los demás: universalismo y benevolencia). Por último, también podría evidenciarse la relación del sexo con las actitudes hacia la inclusión, aunque en este caso la literatura previa no es uniforme (Batsiuo, Bebetsos, Panteli y Antoniou, 2008; Eichinger, Rizzo y Sirotnik, 1991).

\section{Método}

\section{Participantes}

Se adoptó un procedimiento no probabilístico de tipo intencional para seleccionar la muestra. Participaron un total de 315 estudiantes (265 mujeres y 50 hombres) con una edad media de 22.35 años. Los ocho grupos-clase que colaboraron en la investigación fueron los siguientes: un grupo de primer curso y otro de tercero por cada título de grado de magisterio (Educación Infantil: $\mathrm{N}_{1^{\circ}}=43$ y $\mathrm{N}_{3^{\circ}}=39$; y Educación Primaria: $\mathrm{N}_{1^{\circ}}=36$ y $\mathrm{N}_{3^{\circ}}=37$ ), y dos grupos por cada uno de los dos cursos de la Licenciatura en Psicopedagogía $\left(\mathrm{N}_{1^{\circ}}=82\right.$ y $\left.\mathrm{N}_{2^{\circ}}=76\right)$, todos ellos de la Facultad de Ciencias de la Educación de la Universidad de Córdoba. Los datos fueron recogidos durante el curso académico 2012-2013. 


\section{Instrumentos}

Se utilizaron dos instrumentos para la recogida de datos. El primero de ellos fue el Cuestionario de Actitudes hacia la Inclusión Educativa, cuyos primeros ítems cuestionaban sobre datos sociodemográficos, de formación (edad, sexo, titulación, curso), existencia de contacto o experiencia previa con personas con NEE y, en el caso de respuesta positiva a esta cuestión, sobre el tipo de experiencia y la duración de la misma. A continuación, el instrumento presentaba ya los ítems críticos mediante los que se medía la actitud hacia la inclusión. El estudio original a partir del que se adaptaron los ítems críticos corresponde a Boer, Timmerman, Pijl y Minnaert (2012), quienes validaron un instrumento para medir las actitudes de docentes, padres y compañeros hacia la inclusión de alumnado con NEE. Estos autores tomaron como marco teórico la conocida teoría de los tres componentes de la actitud (afectivo, cognitivo y conductual) de Rosenberg y Hovland (1960). La parte del instrumento que adaptamos para nuestra investigación fue el cuestionario dirigido a docentes, sobre el que Boer y sus colegas informan de un coeficiente rho de fiabilidad de .91. Consta de 19 ítems que se agrupan en torno a los tres componentes de la actitud: cognitivo (ítems $1,2,5,8,9,19$ ), afectivo (ítems 3, 6, 11, 12, 14, 15, 17) y conductual (ítems 4, 7, 10, $13,16,18)$. La escala de respuesta fue de cuatro puntos: iba de 1 (totalmente en desacuerdo) a 4 (totalmente de acuerdo). Antes de que los participantes contestaran a cada uno de los ítems, debían leer un caso que reflejaba las características de un niño (Juan) con hiperactividad. Los 17 ítems sobre los que finalmente se computaron las respuestas (dos de ellos restaban fiabilidad al instrumento) se pueden revisar en la primera columna de la tabla 1.

El segundo instrumento utilizado fue el Cuestionario de Valores de Schwartz. Los diez valores, basados en metas motivacionales, se agrupan en dos dimensiones bipolares: apertura al cambio (estimulación y autodirección) vs.conservación (seguridad, conformidad y tradición), y autotrascendencia (universalismo y benevolencia) $v s$. autopromoción (hedonismo, logro y poder). Para este estudio se seleccionó la versión reducida de 21 ítems utilizada por la Encuesta Social Europea. La consistencia interna de este cuestionario en su adaptación al español resulta aceptable, aunque muy ajustada. Zubieta, Fernández y Sosa (2012) informan sobre coeficientes alpha de .65 en autotrascendencia, .64 en conservación, .72 en autopromoción y .65 en apertura. El cuestionario comenzaba con la siguiente pregunta: ¿En qué grado se parece esta persona a ti? A partir de ella, los participantes respondían sobre la semejanza percibida en una escala tipo Likert que iba de 1 (no se parece nada a mí) a 6 (se parece mucho a mí).

\section{Procedimiento}

Una vez seleccionados los instrumentos descritos, así como los grupos-clase a los que se les iba a invitar a participar en la investigación, se procedió a la recolección de los datos de manera presencial en el aula. En cada grupo, tras una breve descripción del documento que contenía ambos cuestionarios, los participantes emitieron sus respuestas sin fijación de un tiempo límite. 
Los datos recogidos fueron procesados con las aplicaciones AMOS (v.20) y SPSS (v.20) en dos fases:1) análisis de validez y fiabilidad del cuestionario de actitudes, para lo que se llevó a cabo un análisis factorial confirmatorio con el objeto de comprobar el ajuste de los datos a modelos previos; un análisis factorial exploratorio con el fin de identificar la estructura empírica de los datos; y un análisis de consistencia interna del instrumento; 2) en la segunda fase se procedió a dar respuesta a los objetivos del estudio, para lo que se caracterizó descriptivamente la muestra (primer objetivo) y posteriormente, con el fin de alcanzar el segundo objetivo y comprobar las hipótesis, se analizó correlacionalmente la asociación de los valores, las variables sociodemográficas, formativas, y de experiencia previa con las actitudes hacia la inclusión, extendiendo los resultados correlacionales mediante pruebas de hipótesis sobre diferencia entre grupos (test $t$, ANOVA, Kruskal-Wallis, $U$ de Mann-Whitney) en el caso de las variables sociodemográficas, formativas y de experiencia previa. Por último, se acometió un análisis predictivo como paso avanzado en el logro del segundo objetivo. En la siguiente sección se informará en primer lugar sobre la calidad del instrumento (fase 1) y, en relación con la fase 2, se presentarán dos apartados. En el primero de ellos se relatarán los resultados descriptivos sobre las variables de experiencia previa, actitudinales y de valores (los correspondientes a las variables sociodemográficas y de formación ya se han presentado al describir la muestra); así como los resultados correlacionales y de prueba de hipótesis. En el segundo apartado se resumirán los resultados de los análisis de regresión.

\section{Resultados}

\section{Validación y fiabilidad del Cuestionario de Actitudes hacia la Inclusión Educativa}

La versión holandesa del cuestionario (Boer et al., 2012) fue adaptada al español, para lo que se procedió a comprobar la estructura unidimensional inicial, así como una segunda estructura tridimensional potencial (componentes cognitivo, afectivo y conductual) mediante análisis factoriales confirmatorios con AMOS, una vez recodificadas las puntuaciones obtenidas en los ítems de sentido inverso (2, 3, 6, 8, 9 y 11). Se utilizó el método de extracción de máxima verosimilitud, no pudiéndose demostrar un ajuste adecuado entre ninguna de las dos estructurasy nuestros datos (en ambos casos, $\chi^{2} / \mathrm{gl}>5$; CFI y GFI $<.95$, RMSEA $>.08$ ).

Dada la ausencia de bondad de ajuste, se procedió a un análisis factorial exploratorio con SPSS, utilizando el mismo método de extracción (máxima verosimilitud). Previamente se eliminaron los ítems 3 y 9 del análisis al comprobar que no contribuían a la fiabilidad del instrumento. Esta se incrementaba hasta .83 tomando los 17 ítems restantes. Por otra parte, la prueba de esfericidad de Bartlett demostró una diferencia significativa entre la matriz empírica de correlaciones y la matriz identidad $\left(\chi^{2}[136]=1585.38, p<.001\right)$, lo que permitió continuar con la extracción de factores. Asimismo, la medida de adecuación muestral de Kaiser-Meyer-Olkin informó sobre un grado notable de varianza común entre los ítems (KMO=.844). 
Se forzó una solución de tres factores, puesto que el criterio del autovalor mayor que 1 arrojaba una solución de cuatro factores con un cuarto componente muy débil. La prueba de bondad de ajuste no permitió rechazar la hipótesis nula $\left(\chi^{2}[88]=107.57\right.$, $p=.077$ ), de lo que se dedujo la adecuación del modelo para explicar la matriz correlacional. La varianza explicada por la solución tridimensional quedó cifrada en el $50 \%$, que se considera limitada, pero suficiente a efectos de los análisis posteriores.

La solución fue rotada inicialmente con Varimax, pero las distribuciones de puntuaciones factoriales resultantes en los factores que se demostraron fiables correlacionaron significativamente, lo que condujo al replanteamiento de la decisión inicial y a la adopción de un método oblicuo de rotación (Oblimin con normalización de Kaiser [delta=0]). El modelo rotado se presenta en la tabla 1 , en la que se muestran las saturaciones de los ítems en los factores.

\begin{tabular}{|c|c|c|c|}
\hline \multirow{2}{*}{ Ítems } & \multicolumn{3}{|c|}{ Factores } \\
\hline & 1 & 2 & 3 \\
\hline $\begin{array}{l}\text { 13. Estoy dispuesto/a a incluir estudiantes como Juan en una clase ordinaria con el } \\
\text { apoyo necesario. }\end{array}$ & .712 & .050 & .078 \\
\hline $\begin{array}{l}\text { 18. Estoy dispuesto/a a adaptar mis habilidades comunicativas para asegurar que } \\
\text { estudiantes como Juan se incorporen de manera normal. }\end{array}$ & .627 & .115 & .025 \\
\hline $\begin{array}{l}\text { 19. Es posible enseñar a niños y niñas con capacidades normales y aquellos otros } \\
\text { con necesidades especiales en la misma clase. }\end{array}$ & .617 & .030 & .033 \\
\hline $\begin{array}{l}\text { 16. Estoy dispuesto/a a modificar el espacio físico del aula para la inclusión de } \\
\text { estudiantes como Juan en clases normales. }\end{array}$ & .583 & .055 & .001 \\
\hline $\begin{array}{l}\text { 1. Estudiantes como Juan tienen derecho a ser educados en la misma clase que el } \\
\text { resto de estudiantes que tienen un desarrollo normal. }\end{array}$ & .560 & .087 & .004 \\
\hline $\begin{array}{l}\text { 5. Se deberían crear todas las oportunidades para que Juan se desarrolle dentro del } \\
\text { grupo-clase. }\end{array}$ & .527 & .053 & .080 \\
\hline $\begin{array}{l}\text { 4. Estoy dispuesto/a a animar a Juan a que participe en todas las actividades sociales } \\
\text { de la clase normal. }\end{array}$ & .507 & .022 & .176 \\
\hline $\begin{array}{l}\text { 10. Estoy dispuesto/a a adaptar el currículum para satisfacer las necesidades de } \\
\text { todos los estudiantes en función de su capacidad. }\end{array}$ & .444 & .131 & .213 \\
\hline $\begin{array}{l}\text { 2. La inclusión NO es una práctica educativa deseable para estudiantes con un } \\
\text { desarrollo normal. }\end{array}$ & .424 & .084 & .062 \\
\hline $\begin{array}{l}\text { 14. Me siento seguro/a cuando llevo a la práctica adaptaciones curriculares } \\
\text { individualizadas. }\end{array}$ & .024 & .868 & .072 \\
\hline $\begin{array}{llllll}\text { 15. Me siento seguro/a cuando corrijo/evalúo adaptaciones curriculares } \\
\text { individualizadas. }\end{array}$ & .086 & .861 & .065 \\
\hline $\begin{array}{|llllllll|}\text { 12. Me siento seguro/a a la hora de diseñar adaptaciones curriculares } \\
\text { individualizadas. }\end{array}$ & .106 & .717 & .222 \\
\hline 17. Tengo confianza en mí mismo/a para manejar comportamientos difíciles. & .233 & .327 & .121 \\
\hline $\begin{array}{l}\text { 11. NO me gustaría adaptar el currículum para satisfacer las necesidades de todos } \\
\text { los estudiantes. }\end{array}$ & .079 & .036 & .809 \\
\hline $\begin{array}{l}\text { 8. Los/as maestros/as de una clase normal NO pueden satisfacer las necesidades de } \\
\text { los estudiantes como Juan. }\end{array}$ & .051 & .101 & .384 \\
\hline
\end{tabular}




\begin{tabular}{|l|c|c|c|}
\hline $\begin{array}{l}\text { 6. Me siento incómodo/a incorporando estudiantes como Juan en una clase normal } \\
\text { con otros estudiantes sin discapacidad. }\end{array}$ & .340 & .028 & $\mathbf{. 3 5 0}$ \\
\hline 7. Estoy dispuesto/a a adaptar los objetivos a cada estudiante. & .257 & .060 & $\mathbf{. 3 3 8}$ \\
\hline
\end{tabular}

Tabla1. Matriz de configuración rotada (saturaciones de las variables en los factores)

Nota. En negrita se destacan las saturaciones más altas de los ítems en cada factor.

Se utilizó el método de regresión para obtener una estimación de las puntuaciones factoriales, verificándose una correlación elevada entre los factores 1 y 3 ( $r=.621$, $p<.001)$, una moderada entre los factores 1 y $2(r=.275, p<.001)$, y otra ligera entre los factores 2 y 3 ( $r=.167, p=.004)$. Los ítems 6 y 7 son los que contribuyen en mayor medida a la alta correlación entre los factores 1 y 3, con saturaciones semejantes en ambos.

Considerando el contenido de los ítems que saturan más en cada factor, se llegó a la siguiente interpretación:

a) Factor 1: Creencias y predisposición comportamental (AG). Es el factor que agrupa un mayor número de ítems: 9 en total. Incluye ítems que hacen referencia a la dimensión cognitiva o de creencias generales positivas hacia la inclusión (1, 2, 5, 19), e ítems que se relacionan con la dimensión comportamental o acciones concretas que el profesional de la educación puede llevar a cabo para transferir el principio de inclusión a la práctica del aula (4, $10,13,16,18)$.

b) Factor 2: Autoconfianza (F1). Agrupa un total de 4 ítems (12, 14, 15 y 17). Todos hacen referencia a la dimensión afectiva, y más concretamente a la confianza y seguridad que el profesional de la educación tiene para gestionar la diversidad mediante el diseño e implementación de actuaciones educativas (adaptaciones curriculares individualizadas, gestión de conductas disruptivas).

c) Factor 3: Actitud positiva hacia la resolución de necesidades individuales (F3).Incluye 4 ítems en los que están representados los tres componentes de la actitud (la afectividad en los ítems 6 y 11; el comportamiento en el ítem 7; y la cognición en el ítem 8). Exceptuando el ítem 6, el resto hacen referencia al principio de individualización de la enseñanza.

Una vez aclarada la estructura del constructo, se procedió al cálculo de la consistencia interna. Los coeficientes de fiabilidad en los tres factores (alpha de Cronbach) fueron .81, .21 y .64, respectivamente. Mientras que la consistencia interna del primer factor fue elevada, la del segundo se alejaba mucho del umbral comúnmente aceptado (en torno a .70), por lo que se rechazó su uso en los análisis críticos. Por su parte, el tercer factor tampoco alcanzó el umbral predeterminado, pero se aproximaba a él y, además, el coeficiente correspondiente se obtuvo a partir de solo cuatro ítems, lo que implica una clara tendencia hacia la consistencia interna si se considera que la fiabilidad correlaciona altamente con el número de ítems.

Los dos factores considerados fiables, el 1 y el 3, son precisamente aquellos entre los que se ha encontrado una correlación notable, validándose así parcialmente la 
conclusión de Boer et al. (2012) sobre la existencia de un único factor general de actitud hacia la inclusión. Considerando la asociación entre los dos factores, los 13 ítems que saturaban en ellos se agregaron para obtener la distribución de puntuaciones sobre la actitud general. Asimismo, se calculó la fiabilidad del conjunto de estos ítems, arrojando el análisis un alpha de .83. Se decidió, por tanto, utilizar estas tres variables dependientes en los análisis posteriores: actitud general hacia la inclusión (AG), creencias y predisposición comportamental (F1), y actitud positiva hacia la resolución de necesidades individuales (F3).

\section{Caracterización actitudinal de la muestra y variables asociadas}

Junto con las variables sociodemográficas y formativas, en las que los participantes ya fueron descritos en la sección metodológica, ha de constatarse asimismo la heterogeneidad de la muestra en relación con el contacto o experiencia previa con personas con NEE: el $43.20 \%$ contaba con ella y el $56.80 \%$ no. Los primeros $(\mathrm{N}=135)$ dijeron haber tomado contacto con personas con NEE principalmente a través de la asignatura de Practicum de los títulos de magisterio y psicopedagogía (71.70\%), y también en ámbitos laborales (32.07\%), familiares (12.26\%) y de actividades de voluntariado (10.38\%). Por otra parte, fueron menos numerosas las personas que respondieron a la duración de la experiencia $(\mathrm{N}=106)$. El mayor número de frecuencias se situó en la categoría "10-52 semanas" (35-85\%), seguida por "más de 52 semanas” (26.41\%), “5-9 semanas” (21.70\%) y “de 1 día a 4 semanas” (16.04\%).

En lo que se refiere a los constructos centrales medidos con los dos instrumentos del estudio, las medias de las tres variables actitudinales son altas (tabla 1). Por consiguiente, junto a la actitud general positiva hacia la inclusión, quedan muy reforzados los dos componentes de la misma (factores 1 y 3). En lo que respecta a los valores, los participantes manifestaron poseer en un alto grado los de apertura al cambio y autotrascendencia; en segundo lugar, puntuaron en niveles medio-altos en valores de conservación y autopromoción, si bien estos no correlacionan significativamente con las actitudes hacia la inclusión. Solo lo hacen los valores de apertura y autotrascendencia.

\begin{tabular}{|c|c|c|c|c|c|c|}
\hline Variables & $M$ & DT & Rango & AG & F1 & F3 \\
\hline 1. Actitud general (AG) & 3.36 & .302 & $1-4$ & - & - & - \\
\hline 2. Creencias y predisposición (F1) & 3.66 & .356 & $1-4$ & - & - & - \\
\hline $\begin{array}{l}\text { 3. Actitudes positivas resolución } \\
\text { necesidades (F3) }\end{array}$ & 3.53 & .490 & $1-4$ & - & - & - \\
\hline 4. Apertura al cambio & 4.74 & .741 & $1-6$ & $.323^{* *}$ & $.336^{* *}$ & $.122^{*}$ \\
\hline 5. Conservación & 3.88 & .776 & $1-6$ & .070 & .057 & .079 \\
\hline 6. Autotrascendencia & 5.36 & .603 & $1-6$ & $.447^{* *}$ & $.502^{* *}$ & $.297^{* *}$ \\
\hline 7. Autopromoción & 3.75 & .801 & $1-6$ & .016 & .018 & -.086 \\
\hline
\end{tabular}

Tabla2. Estadísticos descriptivos y correlaciones producto-momento de Pearson entre los valores y las actitudes $(\mathrm{N}=315) .{ }^{*} p<.05 ;{ }^{* *} p<.01$ 
Junto con las correlaciones de Pearson entre actitudes y valores, se obtuvieron las correlaciones biseriales puntuales del sexo con las actitudes, encontrándose coeficientes significativos, aunque de tamaño reducido, en todos los casos. Concretamente, el sexo correlacionó con AG ( $\left.r_{b p}=.128, p=.023\right)$, F1 $\left(r_{b p}=.165, p<.003\right)$ y F3 $\left(r_{b p}=.122, p=.031\right)$, observándose medias ligeramente más elevadas en las mujeres que en los hombres (véase figura 1). Estas diferencias fueron ratificadas mediante la prueba $t$ de Student en el caso de AG con un tamaño pequeño del efecto $(t[313]=2.29$, $p=.023, d=.259)$ y de $\mathrm{F} 1$ con un tamaño asimismo pequeño $(t[313]=2.95, p=.003$, $d=.333$ ). En el caso de F3 se aplicó la prueba $U$ de Mann-Whitney por no poder verificarse previamente el supuesto de homoscedasticidad en la prueba de Levene ( $F[1$, 313]=3.92, $p=.049$ ), arrojando un efecto tendencialmente significativo de un tamaño muy leve $(U[313]=5660, z=1.67, p=.095, r=.094)$.

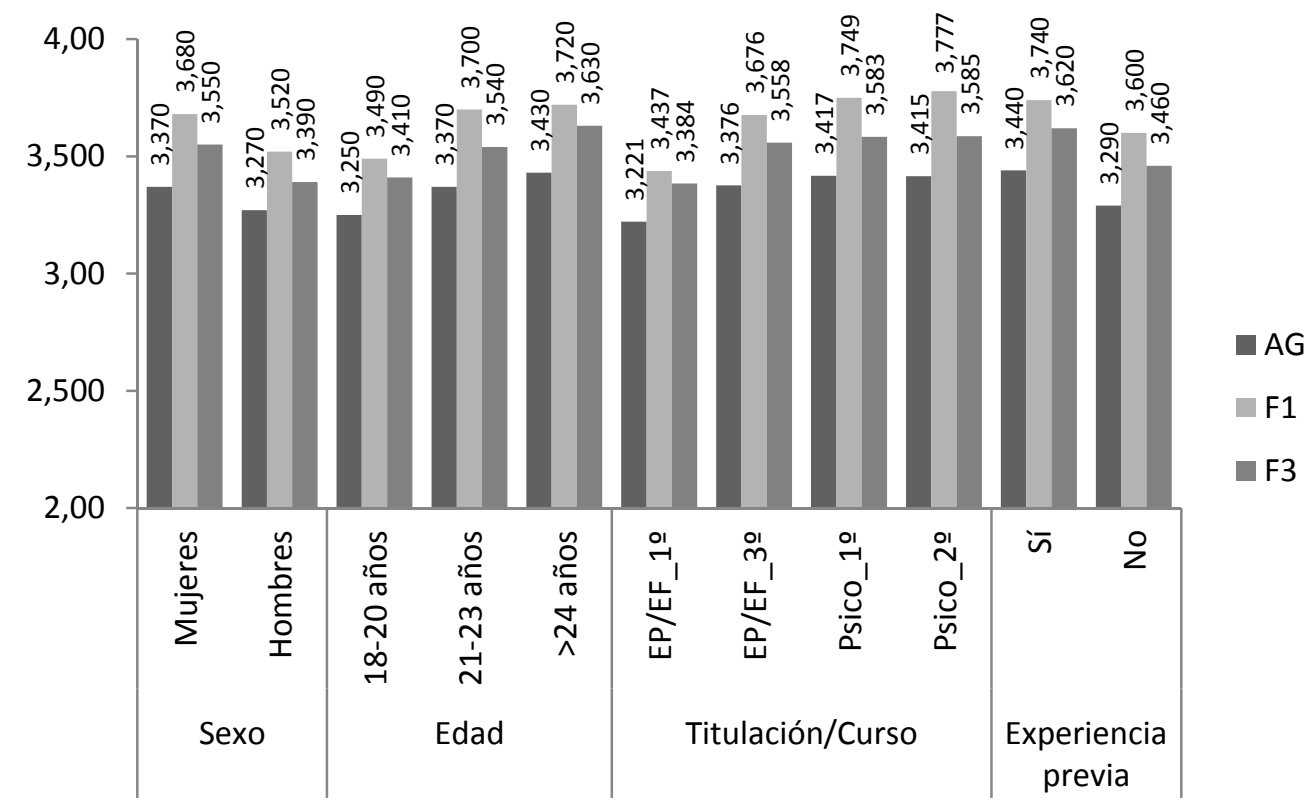

Figura 1. Medias de los grupos de las variables sexo, edad, titulación/curso y experiencia previa.

Posteriormente, se procedió a analizar la relación de la edad y el curso con las actitudes hacia la inclusión (la titulación se analiza conjuntamente con el curso). La primera de ellas correlacionó significativa y positivamente con AG ( $r=.152, p=.007), \mathrm{y}$ con F1 ( $r=.164, p=.004)$, pero no con F3 $(r=.106, p=.061)$. No obstante, se hallaron diferencias significativas entre los grupos al tricotomizar la muestra e implementar un ANOVA en el caso de F3 $\left(F[2,310]=3.28, p=.039, \eta_{p}{ }^{2}=.021\right)$, así como la prueba de Kruskal-Wallis en AG $\left(\chi^{2}[2]=12.15, p=.002\right)$ y F1 $\left(\chi^{2}[2]=20.91, p<.001\right)$. KruskalWallis se aplicó por no poder aceptar el supuesto de homoscedasticidad en la prueba de 
Levene en AG $(F[2,310]=4.57, p=.011)$ y $\mathrm{F} 1(F[2,310]=5.60, p=.004)$. En relación con las diferencias entre los grupos extremos de edad, las pruebas post hoc (Bonferroni en F3, y $U$ en AG y F1) revelaron en todos los casos efectos que rondaban un tamaño mediano, y que indicaban que los participantes de más edad (24 años o más) se caracterizaban por actitudes más positivas que los más jóvenes (18-20 años) (F3: $p=.011, d=.449$; AG: $p=.002, r=.270$; F1: $p<.001, r=.330$ ), mientras que las actitudes de estos fueron asimismo menos positivas que las del grupo intermedio (21-23 años) en AG con un tamaño pequeño del efecto $(p=.011, r=.160)$, y en F1 con un tamaño ligeramente superior $(p<.001, r=.260)$.

Como era de esperar, el curso también correlacionó significativa y positivamente con las tres variables actitudinales: AG $(r=.252, p<.001)$, F1 $(r=.368, p<.001)$ y F3 $(r=.159, p=.005)$. La prueba de Kruskal-Wallis arrojó diferencias significativas entre los cursos evaluados en AG $\left(\chi^{2}[3]=20.07, p<.001\right)$, F1 $\left(\chi^{2}[3]=48.92, p<.001\right)$ y F3 $\left(\chi^{2}[3]=8.45, p=.038\right)$. Como prueba post hoc se implementó $U$ de Mann-Whitney para identificar las diferencias por pares. En todos los casos se encontraron diferencias estadísticamente significativas entre los estudiantes de $1^{\circ}$ y los de $3^{\circ}$ de los títulos de magisterio (AG: $p=.001, r=.257$; F1: $p<.001, r=.338$; F3: $p=.027, r=.177$ ), así como al comparar al alumnado de $1^{\circ}$ de magisterio con el de $1^{\circ}$ de psicopedagogía(AG: $p<.001$, $r=.298$; F1: $p<.001, r=.449$; F3: $p=.010, r=.203)$ y con el de $2^{\circ}$ de esta misma titulación (AG: $p<.001, r=.296$; F1: $p<.001, r=.498$; F3: $p=.050, r=.156$ ).

En lo que se refiere a la existencia de experiencia previa, esta covarió asimismo de forma positiva con las tres variables dependientes: AG $\left(r_{b p}=.254, p<.001\right)$, F1 $\left(r_{b p}=.202, p<.001\right)$ y F3 $\left(r_{b p}=.166, p=.003\right)$. Las medias actitudinales fueron más elevadas en los participantes con experiencia previa con personas con NEE (véase figura 1). Como en el caso de la variable anterior, se verificó la existencia de diferencias estadísticamente significativas entre los grupos mediante $t$ de Student y $U$ de Mann-Whitney. Esta vez, $t$ fue aplicable en AG, verificándose un efecto de tamaño medio $(t[313]=4.57, p<.001, d=.517)$, así como en $\mathrm{F} 3$, evidenciándose un efecto pequeño $(t[313]=2.93, p=.004, d=.354)$. Por su parte, una vez que no se pudo asumir el supuesto de igualdad de varianzas, $U$ sirvió para verificar las diferencias en el caso de F1, hallándose un efecto de tamaño nuevamente pequeño (U[313] $=9061, z=3.91$, $p<.001, r=.220$ ).

Ya únicamente en un sector minoritario de la muestra se recogieron datos sobre la duración de la experiencia previa y el ámbito o tipo de la misma. De manera inesperada, la duración del contacto no alcanzó correlación significativa con ninguna de las variables actitudinales: AG ( $r=.165, p>.05)$; F1 $(r=.125, p>.05)$ y F3 $(r=.108$, $p>$.05). En cuanto al tipo de experiencia, no pudieron verificarse diferencias entre las cuatro categorías comparadas (familia, trabajo, Practicum, voluntariado) en ninguna de las variables de actitud: $\mathrm{AG}(F[3,128]=0.76, p=.519), \mathrm{F} 1\left(\chi^{2}[3]=0.72, p=.869\right)$ y F3 $(F[3,128]=0.32, p=.812)$.

En resumen, los participantes se caracterizan por poseer actitudes muy positivas hacia la inclusión educativa de personas con NEE, si bien se evidencian diferencias muy ligeras a favor de las mujeres, y diferencias de tamaño medio entre los estudiantes mayores y los más jóvenes, así como efectos diferenciales que, en general, oscilan 
entre un tamaño pequeño y medio entre los estudiantes de primer curso y aquellos que se encuentran en tercero de diplomatura y en los cursos de la licenciatura, llegando el efecto a ser de tamaño elevado en F1 cuando se compara a los estudiantes de $1^{\circ}$ de magisterio con los de $2^{\circ}$ de psicopedagogía. Asimismo, se hallaron diferencias que fluctúan, según los factores, entre pequeñas (F1 y F3) y grandes (AG) entre quienes han mantenido contacto previo con personas con NEE y aquellos que carecen de experiencia relacional con ellas.

\section{Predictores actitudinales}

Se sometieron a análisis de regresión lineal los predictores potenciales de cada una de las tres variables actitudinales (es decir, las variables que correlacionaban con ellas, incluidos los valores, no solo los polos dimensionales de Schwartz; únicamente se excluyó el curso porque las pruebas de hipótesis sugerían una relación curvilínea de esta variable con las actitudes: estas mejoraban significativamente en el primer bienio, pero no en el siguiente). En cada uno de los tres análisis (uno por cada variable actitudinal) se utilizó el método de pasos sucesivos. La tabla 3 refleja los resultados del modelo final en cada caso.

\begin{tabular}{llll}
\hline \multirow{2}{*}{ Predictores } & \multicolumn{3}{c}{ Modelos finales } \\
\cline { 2 - 4 } & AG & F1 & F3 \\
\hline Edad & \multicolumn{3}{c}{$.124^{*}$} \\
Sexo & \multicolumn{3}{c}{$.115^{*}$} \\
Experiencia & $.213^{* *}$ & $.130^{* *}$ & $.115^{* *}$ \\
Autodirección & $.195^{* *}$ & $.177^{* *}$ \\
Poder & & & $-.122^{*}$ \\
Autotrascendencia & $.343^{* *}$ & $.390^{* *}$ & $.293^{* *}$ \\
\hline$R^{2}$ & .286 & .334 & .131 \\
\hline
\end{tabular}

Tabla 3. Coeficientes estandarizados $(\beta)$ en los tres modelos finales arrojados por los análisis de regresión lineal. ${ }^{*} p .05 ; * * p<.01$

Se observa que el porcentaje explicado de la varianza en cada modelo no es elevado. Sin embargo, se identifican una serie de predictores significativos. Se destacarían la autotrascendencia y la experiencia como variables predictivas comunes a las tres variables actitudinales. Las variables sociodemográficas de sexo y edad anticipan únicamente la dimensión F1. Esta es precisamente la que es explicada en mayor medida por los predictores incluidos en el modelo (33\% de la varianza).

En general, se constata la coherencia de los resultados correlacionales con los del análisis de regresión. Las correlaciones más elevadas correspondían a la asociación de las actitudes hacia la inclusión educativa con los valores de autotrascendencia, reafirmándose estos vínculos preferentes en los modelos de regresión. La segunda variable estable a través de los modelos, la experiencia previa, correlacionó 
significativa pero débilmente con las actitudes, atribuyéndosele asimismo una capacidad predictiva ligera sobre las tres variables actitudinales. Por su parte, el sexo y la edad, que correlacionaron muy suavemente con las actitudes, mantuvieron su estatus de predictores significativos de F1, pero con coeficientes estandarizados de pequeño tamaño.

\section{Conclusiones}

El estudio evidencia que los estudiantes universitarios de titulaciones de educación mantienen la creencia de que la práctica inclusiva es la mejor forma de educar a todo el alumnado, considerando que se deben crear oportunidades para que todos y todas puedan desarrollarse dentro del grupo-clase junto al resto de sus compañeros. Además, están dispuestos a implementar acciones que faciliten la inclusión de los niños y niñas con NEE como, por ejemplo, flexibilizar el currículum, modificar espacios o adaptar sus habilidades comunicativas, defendiendo que es el docente el que debe ajustarse a la diversidad del aula y no el alumnado con NEE al docente o al currículum. Este es, por cierto, un principio fundamental que debe ser adquirido en la formación inicial (Agencia Europea para el Desarrollo de la Educación del Alumnado con NEE, 2009). Los participantes desarrollan también sentimientos o afectos positivos, ya que tienen la impresión de que se van sentir cómodos llevando a cabo futuras prácticas inclusivas. A pesar de que en el instrumento de recogida de datos se les sugerían posibles dificultades con las que podrían encontrarse en un futuro cuando quisieran poner en práctica la inclusión, los estudiantes universitarios manifiestan actitudes muy positivas, reforzando la validez convergente de resultados similares previamente hallados en el contexto nacional con instrumentos de medida diferentes (Sandoval, 2009; Tárraga et al., 2013).

Junto a la leve ventaja de las mujeres con respecto a los hombres en el tono favorable de sus actitudes hacia la inclusión -coherente con los resultados aportados anteriormente (e.g., Eichinger et al., 1991)-, también se evidencia la capacidad de la formación (Kim, 2011; Swain et al., 2012; Sze, 2009) y de la existencia de experiencia previa (Campbell et al., 2003; Swain et al., 2012) para marcar diferencias actitudinales, en este caso de tamaño medio. Solo se introducirá un matiz por cada una de estas dos últimas variables. En lo que se refiere a la formación inicial, esta resultaría efectiva en un primer periodo, tendiendo después a la estabilización. Se descarta así una relación lineal entre el tiempo de formación y las actitudes. Costello y Boyle (2013) encontraron también resultados diferenciados por etapas. En su muestra, las actitudes progresaron negativamente durante el grado, pero luego se suavizaron considerablemente en el postgrado, difiriendo significativamente entre ambas etapas a favor del postgrado. A la vista de estas disparidades entre etapas, y también entre estudios, habría que analizar en profundidad el currículum formativo de cada contexto para encontrar en él posibles explicaciones (Kim, 2011), sin perjuicio del estudio que asimismo merecerían otras variables individuales o contextuales (por ejemplo, de tipo organizativo) que también condicionan la evolución actitudinal (McCollow et al., 2015). 
En lo que se refiere a la experiencia previa con personas con NEE, la mera existencia de la misma funcionó, aunque débilmente, en el sentido previsto en la literatura psicosocial que asocia el contacto a las actitudes de prejuicio (Allport, 1954; Hewstone y Swart, 2011; Pettigrew y Tropp, 2011), así como en investigaciones de carácter educativo ya mencionadas, tanto sobre profesorado en ejercicio (Ahmmed et al., 2012; Avramidis et al., 2000), como sobre futuros profesores (Campbell et al., 2003; Swain et al., 2012). Aun así, no se pudo confirmar una de las predicciones psicosociales de la hipótesis del contacto: la referida a la asociación positiva de la duración de la experiencia con las actitudes. Por una parte, no siempre se ha observado una relación directa entre contacto y actitudes en profesorado en formación (Crowson y Brandes, 2014), pero además en nuestro estudio se podría suponer la operatividad de un efecto techo: la inmensa mayoría de los participantes se agruparon en la parte alta de la escala de medida de las actitudes, lo que dificultó el hallazgo de diferencias estadísticamente significativas entre los niveles de la variable de agrupamiento. Este hecho también habría incidido en el tamaño reducido de las correlaciones y coeficientes de regresión que asociaron la existencia de experiencia a las actitudes, pero habría afectado más a la duración de la experiencia previa a causa del menor tamaño muestral, con la consiguiente mayor dificultad para alcanzar significación estadística.

En cuanto al tipo de ámbito experiencial o de contacto, y aunque no fuera posible realizar un contraste eficaz debido a las frecuencias pequeñas en la mayoría de los niveles de la variable, se destacará un dato descriptivo de enorme relevancia: más de la mitad de los estudiantes que dijeron haber tenido algún tipo de experiencia previa con personas con NEE identificaron el ámbito del Practicum como escenario del contacto. Por tanto, el Practicum, que es un contexto idóneo para la evolución del pensamiento práctico (Pérez Gómez, 2010), es reconocido como un espacio curricular en el que pueden desarrollarse de manera eficaz actitudes positivas hacia la inclusión (Swain et al., 2012).

En relación con los valores que caracterizan al alumnado de las titulaciones estudiadas, se afirmaría que estos son abiertos, se preocupan por el bienestar de las personas, buscan la igualdad entre ellas, son comprensivos y creativos, les atrae la novedad y los desafíos, se resisten a aceptar las costumbres impuestas, no se conforman con aquello con lo que no están de acuerdo y van más allá del prestigio social. Se trata de valores que se agrupan en los polos dimensionales de autotrascendencia y apertura, que en el análisis correlacional demostraron su asociación con las actitudes en niveles moderados, manteniéndose este mismo tamaño de efecto en el caso de la autotrascendencia como predictor de las tres variables actitudinales analizadas. Por consiguiente, queda refrendada la relación entre el apoyo a la inclusión y los valores de justicia, igualdad y colaboración (Sandoval, 2009), en la medida en que estos son componentes del universalismo y la benevolencia -valores correspondientes al polo dimensional de la autotrascendencia- en la clasificación de Schwartz (1992, 2012).

Para finalizar, introduciremos dos apuntes normativos relacionados con la formación inicial, que se derivan de las conclusiones del estudio. En primer lugar, se 
ha puesto de manifiesto que la etapa de desarrollo profesional inicial es relevante para la evolución de las actitudes hacia la inclusión de alumnado con NEE y, si se combina este factor con el de la experiencia previa, se deduce inmediatamente la necesidad de insistir aún más en la contribución del Practicum al desarrollo actitudinal. En segundo lugar, no se trataría únicamente de facilitar el aprendizaje de competencias actitudinales, sino también de los predictores de las mismas, que se configurarían asimismo como competencias deseables en el desarrollo profesional inicial. Particularmente, los valores de autotrascendencia (universalismo, benevolencia) y, en menor medida, los de apertura al cambio (estimulación, autodirección) resultan fundamentales en una profesión orientada por la ética del cuidado y por los principios de justicia social y respeto a la diversidad.

\section{Referencias bibliográficas}

AGENCIA EUROPEA PARA EL DESARROLLO DE LA EDUCACIÓN DEL ALUMNADO CON NECESIDADES EDUCATIVAS ESPECIALES (2009). Principios fundamentales para la promoción de la calidad de la educación inclusiva. Recuperado de http://www.europeanagency.org/publications/ereports/key-principles-for-promoting-quality-in-inclusiveeducation/key-principles-ES.pdf

AGENCIA EUROPEA PARA EL DESARROLLO DE LA EDUCACIÓN DEL ALUMNADO CON NECESIDADES EDUCATIVAS ESPECIALES (2010). Teacher education for inclusion. International Literature Review. Recuperado de http://www.european-agency.org/sites/default/files/te4i-international-literaturereview_TE4I-Literature-Review.pdf

AHMMED, M., SHARMA, U. y DEPPELER, J. (2012).Variables affecting teachers' attitudes towards inclusive education in Bangladesh. Journal of Research in Special Education Needs, 12 (3), 132-140. doi: 10.1111/j.1471-3802.2011.01226.x

ALLPORT, G. (1954). The nature of prejudice. Cambridge, Mass.: Addison-Wesley.

AVRAMIDIS, E., BAYLISS, P. y BURDEN, R. (2000). A survey into mainstream teachers' attitudes towards the inclusion of children with special educational needs in the ordinary school in one local authority. Educational Psychology, 20 (2), 191211.

AVRAMIDIS, E. y NORWICH, B. (2002). Teachers' attitudes towards integration / inclusion: a review of the literature. European Journal of Special Needs Education, 17 (2), 129-147. doi: 10.1080/08856250210129056

BASABE, N., VALENCIA, J. y BOBOWIK, M. (2011). Valores y actitudes: cultura de violencia y paz. En D. Páez, C. Martín, J, L. González, N. Basabe y J. de Rivera (Eds.), Superando la violencia colectiva y construyendo cultura de paz (pp. 71103). Madrid: Fundamentos.

BATSIOU, S., BEBETSOS, E., PANTELI, P. y ANTONIOU, P. (2008). Attitudes and intention of Greek and Cypriot primary education towards teaching pupils with 
special educational needs in mainstream schools. International Journal of Inclusive Education, 12 (2), 201-219. doi: 10.1080/13603110600855739

BOER, A. DE, PIJL, S. J. y MINNAERT, A. E. (2011). Regular primary school teacher attitudes towards inclusive education: a review of the literature. International Journal of Inclusive Education, 15 (3), 331-353. doi: 10.1080/13603110903030089

BOER, A. DE, TIMMERMAN, M., PIJL, J. y MINNAERT, A. (2012). The psychometric evaluation of a questionnaire to measure attitudes towards inclusive education. European Journal of Psychology of Education, 27 (4), 573-589. doi: 10.1007/s10212-011-0096-z

CAMPBELL, J., GILMORE, L. y CUSKELLY, M. (2003). Changing student teachers' attitudes towards disability and inclusion. Journal of Intellectual \& Developmental Disability, 28 (4), 369-379. doi:10.1080/13668250310001616407

CHINER, E. (2011). Las percepciones y actitudes del profesorado hacia la inclusión del alumnado con necesidades educativas especiales como indicadores del uso de prácticas educativas inclusivas en el aula. Tesis doctoral, Universidad de Alicante. Recuperado de http://rua.ua.es/dspace/bitstream/10045/19467/1/Tesis_Chiner.pdf

COSTELLO, S. y BOYLE, C. (2013). Pre-service secondary teachers' attitudes towards inclusive education. Australian Journal of Teacher Education, 38 (4), 129143. doi: 10.14221/ajte.2013v38n4.8

CROWSON, H. M. y BRANDES, J. A. (2014). Predicting pre-service teachers' opposition to inclusion of students with disabilities: a path analytic study. Social Psychology of Education, 17 (1), 161-178. doi: 10.1007/s11218-013-9238-2

EICHINGER, J., RIZZO, T. y SIROTNIK, B. (1991). Changing attitudes toward people with disabilities. Teacher Education and Special Education, 14(2), 121-126. doi:10.1177/088840649101400207

FORLIN, C., SHARMA, U. y LOREMAN, T. (2007). An international comparison of pre-service teacher attitudes towards inclusive education. Disabilities Studies Quarterly, 27 (4). Recuperado de http://dsq-sds.org/article/view/53/53

GALLEGO, J. L. y RODRÍGUEZ, A. (2012). ¿Cómo aprenden ahora los estudiantes de magisterio a atender a la diversidad de su alumnado? Revista de Currículum y Formación del Profesorado, 16 (2), 327-342.

GAO, W. y MAGER, G. (2011). Enhancing preservice teachers' sense of efficacy and attitudes toward school diversity through preparation: A case of one US inclusive teacher education program. International Journal of Special Education, 26 (2), 92107.

HEWSTONE, M. y SWART, H. (2011). Fifty-odd years of inter-group contact: From hypothesis to integrated theory. British Journal of Social Psychology, 50 (3), 374386. doi: 10.1111/j.2044-8309.2011.02047.x 
HWANG, Y. y EVANS, D. (2011). Attitudes towards inclusion: Gaps between belief and practice. International Journal of Special Education, 26 (1), 136-146.

KIM, J. (2011). Influence of teacher preparation programmes on preservice teachers' attitudes toward inclusion. International Journal of Inclusive Education, 15 (3), 355-377. doi: 10.1080/13603110903030097

LOREMAN, T., SHARMA, U. y FORLIN, C. (2013). Do pre-service teachers feel ready to teach in inclusive classrooms? A four country study of teaching selfefficacy. Australian Journal of Teacher Education, 38 (1), 27-44. doi: 10.14221/ajte.2013v38n1.10

LUQUE, D. J. y LUQUE-ROJAS, M. J. (2011). Conocimiento de la discapacidad y relaciones sociales en el aula inclusiva. Sugerencias para la acción tutorial. Revista Iberoamericana de Educación, 54 (6), 1-12.

MANGANO, M. (2015). Teacher views on working with others to promote inclusion. En D. Chambers (Ed.), Working with Teaching Assistants and Other Support Staff for Inclusive Education (International Perspectives on Inclusive Education) (Vol. 4, pp. 117-132). Bingley, UK: Emerald Group Publishing Limited. doi: 10.1108/S1479-363620150000004005

MCCOLLOW, M. M., SHURR, J. y JASPER, A. D. (2015). Best practices in teacher training and professional development for including learners with low-incidence disabilities. En E. A. West (Ed.), Including Learners with Low-Incidence Disabilities (International Perspectives on Inclusive Education) (Vol. 5, pp. 37-62). Bingley, UK: Emerald Group Publishing Limited. doi: 10.1108/S1479363620140000005002

MONSEN, J. J., EWING, D. L. y KWOKA, M. (2014). Teachers' attitudes towards inclusion, perceived adequacy of support and classroom learning environment. Learning Environments Research, 17 (1), 113-126. doi: 10.1007/s10984-013-91448

PÉREZ GÓMEZ, A. (2010). Aprender a educar. Nuevos desafíos para la formación de docentes. Revista Interuniversitaria de Formación de Profesorado, 24 (2), 37-60.

PETTIGREW, T. F. y TROPP, L. R. (2011). When groups meet: The dynamics of intergroup contact. Philadelphia, PA: Psychology Press.

POLO, T., FERNÁNDEZ, C. y DÍAZ, C. (2011). Estudio de las actitudes de estudiantes de Ciencias Sociales y Psicología: relevancia de la información y contacto con personas discapacitadas. Universitas Psychologica, 10 (1), 113-123.

RODRÍGUEZ, E. ETOPA, P. y RODRÍGUEZ, A (2002). Las actitudes de los futuros maestros y maestras de Educación Infantil hacia la discapacidad: un factor de prevención. El Guiniguada, 11, 171-180.

ROSENBERG, M. J. y HOVLAND, C. I. (1960). Cognitive, affective and behavioral components of attitudes. En C. I. Hovland, y M. J. Rosenberg (Eds.), Attitude, Organization, and Change. New Haven: Yale University Press. 
SANDOVAL, M. (2009). Concepciones de los estudiantes de magisterio sobre la inclusión educativa. Aula abierta, 37 (1), 79-88.

SAVOLAINEN, H., ENGELBRECHT, P., MIRNA, M. y MALINEN, O. (2012). Understanding teachers' attitudes and self-efficacy in inclusive education: Implications for pre-service and in-service teacher education. European Journal of Special Needs Education, 27 (1), 51-68. doi: 10.1080/08856257.2011.613603

SCHWARTZ, S. H. (1992). Universals in the content and structure of values: Theoretical advances and empirical tests in 20 countries. Advances in Experimental Social Psychology, 25, 1-65.

SCHWARTZ, S. H. (2012). An overview of the Schwartz Theory of Basic Values. Online Readings in Psychology and Culture, 2 (1). Recuperado de http://dx.doi.org/10.9707/2307-0919.1116

SELESHO, J. (2012). Inclusion of high school learners in the mainstream: Ecological niche. Anthropologist, 14 (6), 539-543.

SZE, S. (2009). A literature review: Pre-Service teachers' attitudes toward students with disabilities. Education, 130 (1), 53-56.

SWAIN, K. D., NORDNESS. P. D. y LEADER-JANSSEN, E. M. (2012). Changes in preservice teacher attitudes toward inclusion. Preventing School Failure, 56 (2), 7581. doi:10.1080/1045988X.2011.565386

TAIT, K. y PURDIE, N. (2000). Attitudes toward disability: teacher education for inclusive environments in an Australian university. International Journal of Disability, Development and Education, 47 (1), 25-38. doi: 10.1080/103491200116110

TÁRRAGA, R.,GRAU, C. y PEIRAT, J. (2013). Actitudes de los estudiantes del Grado de Magisterio y del Máster de Educación Especial hacia la inclusión educativa. Revista Electrónica Interuniversitaria de Formación del Profesorado, 16 (1). Recuperado de http://revistas.um.es/reifop/article/view/179441

TAYLOR, R. y RINGLABEN, R. (2012). Impacting pre-service teachers' attitudes toward inclusion. Higher Education Studies, 2 (3), 16-23.doi: 10.5539/hes.v2n3p16

ZUBIETA, E., FERNÁNDEZ, O. y SOSA, F. (2012). Bienestar, valores y variables asociadas. Boletín de Psicología, 106, 7-27. 


\section{Correspondencia con los autores}

José Luis ÁLVAREZ CASTILLO

Departamento de Educación

Facultad Ciencias de la Educación

Universidad de Córdoba

Avda. San Alberto Magno s/n

14071 Córdoba

e-mail: jlalvarez@uco.es

Mariana BUENESTADO FERNÁNDEZ

Departamento de Educación

Facultad Ciencias de la Educación

Universidad de Córdoba

Avda. San Alberto Magno s/n

14071 Córdoba

e-mail: m62bufem@uco.es 